\title{
Differential diagnosis of back pain in adult scoliosis (non operated patients)
}

\author{
Manuel Rigo \\ From 7th International Conference on Conservative Management of Spinal Deformities \\ Montreal, Canada. 20-22 May 2010
}

The relationship between back pain and scoliosis is not clear. Old long-term follow-up studies reported no greater incidence or degree of back pain in adult scoliosis than in normal population when matched for age and sex. At the end of the seventies studies were contradictories. While Fowles et al (1978) concluded that untreated scoliosis could be considered as a disabling disease in the adult particularly after the age of 30 , Nachemson (1979) reported a minimal risk of disabling back pain in adult patients with lumbar curves stating that 'whether severe low back pain occurs more often in adults who have lumbar curves than in those whose spines are straight is open to question'. Later, in 1981, Weinstein et al found that backache was more common in scoliosis patients than in the general population with no relation between back pain and the presence of osteoarthritic changes. Kostuik and Ventivoglio (1981) reported a similar incidence of low back pain (59\%) in subjects with lumbar or thoracolumbar curves and normal population. However, they found a correlation between back pain and severity of the curve especially for curves of more than $45^{\circ}$, while patients with no pain tended to have smaller curves. Jackson et al (1983) confirmed that incidence was comparable between adult scoliosis and general population but severity was greater in scoliotic patients. They found that patients with lumbar curve had more pain. Compensatory lumbosacral curves were most painful and disabling. The controversy about how well or bad scoliosis population do it (pain and function) during adult life still continued during the nineties and while the results from the Ste-Justine Adolescent Idiopathic Scoliosis Cohort Study suggested that back pain is responsible for a considerable amount of disability and handicap in later life, the revisited sample from Weinstein et al, showed that untreated late onset

Institut Elena Salvá, Barcelona, Spain

Full list of author information is available at the end of the article idiopathic scoliosis causes little physical impairment other than back pain and cosmetic concerns, with these patients being productive and functional at high level at 50-year follow up. In agreement with the Ste-Justine's study, Schwab F et al (2003) studied the effect of scoliosis in the self-perception of health and showed that patients in this study averaged scores (SF-36) much lower than the norm from both the general US population in all 8 categories and the US population for ages 55-64 in 7 out of 8 categories of the SF-36 questionnaire. Whether or not idiopathic scoliosis developed during growth will alter health related quality of life later and how conservative and/or surgical treatment will change its natural history is something needing further research, however, from a practical point of view, here the question is not that but how scoliosis condition affects the diagnose, prognosis and management of adult patients suffering from chronic back pain.

Adult patients attending our institution - a scoliosisspecific rehabilitation centre - could be divided in two main groups: Group I: Patients attending the clinic with a clear self-conscience of belonging to the scoliosis population because they were mostly diagnosed during childhood or during adolescence - treated or not treated-; Group II: Patients belonging to the back pain population referred to us by their doctors, mostly because they showed a bad response to general rehabilitation and such a bad response was related to a non previously diagnosed scoliosis condition. Generally speaking we could identify patients with idiopathic, congenital and secondary scoliosis in Group I while most of the patients in Group II have developed de novo degenerative scoliosis or scoliosis secondary to any pelvic or lower limbs biomechanical disturbance. The main reasons for consultation in Group I are: documented curve progression, cosmesis, chronic back pain and disability, and prevention in patients that have been followed-up 
during adult life (breathing impairment does not constitute a common primary reason for consultation in our experience). The same reasons can be identified in some patients with no follow-up since they reached bone maturity but when curve progression is the reason for consultation this would be just suspected or selfreported. In this Group I we find two sub-groups of patients self-reporting 1) 'to do it well' or 2) 'to do it bad', in relation with back pain and function. For those 'doing bad' the main reason for that use to be their 'scoliotic condition' with no doubt. We have no a clear idea about how emotional, psychological and cultural influence patient's perception of health in relation to their scoliosis. In Group II, obviously, $100 \%$ of patient selfreport 'to do it bad'. In the sub-group of patients 'doing well' as well as in the sub-group of patients 'doing bad' we can find young and old adults, male and female, mild and severe scoliosis, any curve pattern, treated and non treated scoliosis. It would seem that the diagnose of scoliosis, gender, magnitude of the curve, pattern, etc is not predicting whether a patient will be classified in one or other sub-group. This is obviously just a perception, but it is in agreement with some consistent evidence. For example, Hill et al (2008) found that baseline scoliosis deformity parameters of largest curve size, decompensation in the frontal plane and trunk twist did not predict scoliosis-related pain in young adults. On the other hand Bess et al (2009) concluded that age, comorbidities and sagittal balance did not influence treatment modality for adult scoliosis. They found that young adults went under surgery due to the increase of their frontal plane deformity, while older patients did it due to pain and disability, independently of the radiographic measurements. Final conclusion from this authors was that adult scoliosis patients do not become uniformly disabled with age and that disability can not be solely predicted by radiographic findings. Conclusions could be different just by considering those patients suffering back pain and analysing the relationship between selfreported pain/disability and different radiographic parameters. In order to establish a differential diagnose, prognosis and evidence based treatment plan we should consider only those patients affected with back pain and presenting with scoliosis. For example, rotatory olisthesis did not correlate with pain/disability when sample included adult scoliosis patients with and without pain (Trammell TR et al 1988) but did it when sample included only adult scoliosis patients with pain (Jackson RP et al 1989; Schwab F et al 2006 and Ploumis A et al 2009). Back pain is a multifactorial condition which affects general population. According to Winter et al (1988) adult patients with scoliosis often have back pain but the pain may or not be related to the scoliosis. They concluded that 'a carefully history, physical examinations, routine radiographic examination and on some occasions specialized radiographs, CT, Myelography, discography and facet joint injections will help the physician or surgeon separate out those patients syndromes owing to the curvature versus those not owing to the curvature'. Myelography and discography may have lost their relevance versus MRI. Early studies conducted in painful adult scoliosis patients found fractional lumbosacral curves most painful and disabling (Jackson RP et al 1989). Scoliosis greater than 40 and kyphosis greater than $50^{\circ}$ correlated with increasing pain. They found rotation to be the highest correlated with pain. As a whole these authors found a relationship between pain and rotation, olisthesis, disc degeneration - which was more marked in the mild and lower lumbar spine in combination with thoracolumbar, lumbar and lumbosacral curves - kyphosis $\left(>50^{\circ}\right)$ and curve magnitude $\left(>40^{\circ}\right)$. Later studies (Schwab FJ et al 2002) showed that lateral vertebral olisthesis, L3 and L4 endplate obliquity angles, lumbar lordosis (hypolordosis) and thoracolumbar kyphosis were significantly correlated with pain. These last authors included in their analysis adult scoliosis patients with a previous diagnose of adolescent idiopathic scoliosis as well as adult scoliosis patients with de novo degenerative scoliosis. Patients with idiopathic scoliosis had larger major scoliosis curvatures in comparison with patients diagnosed with de novo degenerative scoliosis although the radiographic parameters correlating with pain were identical for these groups. The authors opinion is that this 'appears to substantiate the belief that a common end pathway (degenerative instability and unfavourable lumbar vertebral alignment) among both groups of patients is related to symptoms rather than the degree of curvature or the cause of the original scoliosis'. In consistency with this, Buttermann GR and Mullin WJ (2008) have reported a correlation between pain/disability and disc degeneration in scoliosis patients. They found that the adult scoliosis patients had pain/disability similar to those of surgical degenerative disc disease control group without scoliosis. Disc degeneration and disc herniation were not related to pain. Those patients with scoliosis and pain which typically occurring at the apex of the scoliosis or at the lumbosacral junction had a significantly higher frequency of inflammatory and plate changes on MRI that did controls. Their conclusion was that 'adult scoliosis patients have multifactorial pain of which one component may be related to degeneration of the lower discs similar to that in nondeformity patients. Additionally, adults scoliosis patients may have MRI findings consistent with discogenic pain at the apex of their curvatures, most commonly at the proximal lumbar levels'. Following their early findings (2002), Schwab F et al (2005) proposed a lumbar classification of scoliosis in 
the adult patient, as a preliminary approach. Patients were classified into one of three types of deformity based on the degree of lordosis (L1-S1) and frontal plane end plate obliquity of L3 on standing radiographs. Type I $=$ lordosis $>50^{\circ}$, L3 obliquity $<15^{\circ}$; Type II = lordosis $35^{\circ}-55^{\circ}$, L3 obliquity $15^{\circ}-25^{\circ}$ and Type III = lordosis $<35^{\circ}$, L3 obliquity $>25^{\circ}$. With increased type (from I to III) self-reported pain and disability increased. Schwab F et al developed a further Clinical Impact Classification of Scoliosis in the Adult (2006) based on previous data which revealed that lumbar lordosis and frontal plane obliquity of L3, but not the Cobb angle, correlated with back pain, while deformity apex and intervertebral subluxation correlated with disability. They used deformity apex, lordosis (L1-S1) and intervertebral subluxation to classify the patients. These authors found that in thoracolumbar and lumbar scoliosis, the loss of lordosis/higher subluxation was associated with lower SRS pain/function and higher Oswestry Disability Index Scores. Lower apex combined with lower lordosis led to higher disability. The Schwab et al clinical impact classification has shown correlation with self-reported disability as well as rates of operative treatment. The Adult Spinal Deformity Committee of the SRS has developed a further classification for adult spinal deformity 'to provide an organizational structure for spinal deformity that permits comparison of similar cases, and provides a framework for an evidence-based approach to operative and nonoperative management of deformity in the adult'. The classification uses Primary curve types according to SRS definition of regions, in combination with adult spinal deformity modifiers: 1) Regional sagittal modifier, 2) Lumbar degenerative modifier, 3) Global balance modifier. The lector can find the original description of this classification (Lowe $\mathrm{T}$, Berven $\mathrm{SH}$, Schwab FJ and Bridwell KH: The SRS Classification for Adult Spinal Deformity. Spine 2006, 31(19) Suppl:S119S125).

In conclusion, it is not possible to predict health related quality of life in adults from their baseline preadult condition. Back pain is multifactorial and lumbar scoliosis could constitute a subgroup within the low back pain population (Pérennou D et al 1994). Adults with painful scoliosis need a complete clinical history, physical exploration and complementary explorations in order to establish whether their pain is related to the scoliosis or not. When pain/disability is present, some radiological parameters correlate with self-reported pain/disability and assessment of those parameters is essential to establish the relationship: deformity level and curve type according to the SRS classification for adult spinal deformity (including idiopathic, de novo degenerative and primary sagittal plane deformity), frontal and sagittal olisthesis, lumbar lordosis (L1-S1 hypolordosis), thoracolumbar kyphosis (T11-L1), end plate L3 obliquity (in relation with a fractional lumbosacral curve), rotation, MRI degenerative changes at the apical level and junctional zones and global frontal and sagittal imbalance. Cobb angle (curve magnitude) does not correlate with back pain although those patients without pain use to present smaller curves and in some studies incidence and degree of back pain was higher in more severe curves $\left(>40^{\circ}-45^{\circ}\right)$. Age did not correlate with pain although pain /disability is the main reason to go under surgery in older adult scoliosis patients. Pain/ disability can be assessed by using VAS, SRS questionnaires and SF-36.

Published: 10 September 2010

doi:10.1186/1748-7161-5-S1-O44

Cite this article as: Rigo: Differential diagnosis of back pain in adult scoliosis (non operated patients). Scoliosis 2010 5(Suppl 1):O44.
Submit your next manuscript to BioMed Central and take full advantage of:

- Convenient online submission

- Thorough peer review

- No space constraints or color figure charges

- Immediate publication on acceptance

- Inclusion in PubMed, CAS, Scopus and Google Scholar

- Research which is freely available for redistribution

Submit your manuscript at www.biomedcentral.com/submit 